\title{
L'impact des Activités Physiques Etsportives dans le Développement Collectif de Certaines Compétences Mentales Chez les Sportifs de Haut Niveau
}

\author{
Dr. Saidia Houari, \\ Directeur du Laboratoire de mesure et d'évaluation des activités sportives, \\ Centre universitaire de Tissemsilt, Algérie \\ Dr. Nahal Hamid, \\ Chef uni d'unité de recherche, Centre universitaire Tissemsilt, ALG
}

Doi: 10.19044/esj.2019.v15n3p276 URL:http://dx.doi.org/10.19044/esj.2019.v15n3p276

\section{Résumé}

L'activité physique est l'une des activités publiques les plus importantes des programmes éducatifs visant à préparer l'individu à être utile à la société. Ce type d'activités constituent la base de la santé du corps humain et de la sécurité de l'esprit. De la même manière, le sport a une place essentielle dans le soin des membres de la société. Il joue également un rôle important dans l'éducation d'un individu à tous les stades, étant l'étape intermédiaire celle qui est la plus importante de l'éducation grâce à l' enthousiasme que démontre l'individu au cours de son développement social, physique, mental et psychologique. Dans le programme d'enseignement sur l'éducation physique et sportive, nous avons constaté que les activités sportives de groupe attirent beaucoup l'attention des adolescents. Ceci est attribué aux implications positives pour eux, ainsi qu'à leur contribution à la satisfaction de leurs besoins et de leurs désirs. Ainsi, cela explique leur intérêt pour eux et les aide à répondre à plusieurs de leurs besoins individuels. L'existence du groupe dans ces activités, qui à leur tour conduisent au développement des compétences mentales et de l'esprit de sport chez l'élève, entraîne AINSI beaucoup de hautes qualités éducatives et morales.

Mots clés: Activités physiques et sportives, aptitudes mentales, athlètes de haut niveau 


\title{
The Impact of Physical and Sports Activities in the Collective Development of Certain Mental Skills Among High Level Athletes
}

\author{
Dr. Saidia Houari, \\ Director of the Laboratory for the Measurement and Evaluation of Sports \\ Activities, University Center of Tissemsilt, Algeria \\ Dr. Nahal Hamid, \\ United Head of Research Unit, University Center Tissemsilt, ALG
}

\begin{abstract}
Physical activity is one of the most important public activities in educational programs that aim to prepare the individual for a society that is useful, and sports activities are the most important physical activities and sports are the basis for health of objects and security of minds, but its place in the care of members of society and education is therefore that it goes hand in hand with the movement of education at all stages, and can -being the most important stages of education is the intermediate stage, which is an enthusiasm passes through the individual during social and physical, mental and psychological development. In the Physical Education and Sport Teaching Curriculum, we find that group sports activities take a great deal of attention from adolescents, considering the positive implications for them, as well as their contribution to meeting their needs. and desires, which explains their interest in them, indicating that they meet many of their individual needs. The existence of the group in these activities, which in turn lead to the development of the student's mental skills in the spirit of the sport, which in turn gain a lot of high educational and moral qualities.
\end{abstract}

Keywords: Physical and sports activities, mental skills, high level athletes

\section{1 - Introduction et problématique:}

L'activité physique est l'une des plus importantes activités publiques dans les programmes éducatifs qui visent à préparer l'individu à une société qui est utile, et les activités sportives sont des activités physiques les plus importantes et les sports sont à la base pour la santé des objets et la sécurité des esprits, mais sa place dans la prise en charge des membres de la société et de l'éducation est donc qu'il va de pair avec le mouvement de l'éducation à tous les stades, et peut-être les étapes les plus importantes de l'éducation est 
l'étape intermédiaire, qui est un enthousiasme passe par la individu au cours du développement social et physique, mental et psychologique (alawi.1995).

Dans le programme d'enseignement de l'éducation physique et du sport, nous constatons que les activités sportives collectives prennent une grande surface d'attention aux adolescents, compte tenu des implications positives pour eux, ainsi que leur contribution à la satisfaction de leurs besoins et désirs, ce qui explique leur intérêt en eux, ce qui indique qu'ils répondent à beaucoup de leurs besoins individuels.

L'existence du groupe dans ces activités, qui à son tour conduire au développement des compétences mentales de l'étudiant dans l'esprit du sport, qui à son tour gagner beaucoup de qualités éducatives et morales élevées (Osaka Rateb ,2001).

Dans cette recherche, nous allons explorer l'impact de l'activité physique sportive collective sur le développement de certaines compétences mentales chez les élèves du secondaire.

En ce sens, nous posons la question suivante:

\section{2-La question générale:}

Y a-t-il des différences statistiquement significatives entre le pré-test et le post-test pour développer certaines compétences mentales dans les activités sportives physiques des élèves intermédiaires?

\section{Questions partielles}

- Y a-t-il des différences statistiquement significatives entre le pré-test et le post-test pour développer la concentration de l'attention dans les activités sportives physiques des élèves intermédiaires?

- Y a-t-il des différences statistiquement significatives entre le pré-test et le post-test dans le développement de la perception mentale dans les activités sportives physiques des collégiens?

- Y a-t-il des différences statistiquement significatives entre le pré-test et le post-test dans le développement de la capacité à se détendre dans les activités sportives physiques des collégiens?

- Y a-t-il des différences statistiquement significatives entre le pré-test et le post-test dans le développement de la capacité à faire face à l'anxiété dans les activités sportives physiques des collégiens?

- Y a-t-il des différences statistiquement significatives entre le pré-test et le post-test dans le développement de la motivation pour la réussite sportive dans les activités sportives physiques des collégiens?

- Y a-t-il des différences statistiquement significatives entre le pré-test et le post-test dans le développement de la capacité à faire face aux activités sportives physiques des collégiens? 


\section{Hypothèses:}

3-1-Hypothèse générale:

Il existe des différences statistiquement significatives entre le pré-test et le post-test dans le développement de certaines compétences mentales dans les activités sportives physiques des collégiens.

\section{2-Hypothèse partielle:}

- Il existe des différences statistiquement significatives entre le pré-test et le post-test pour développer la concentration de l'attention dans les activités sportives physiques collectives chez les collégiens.

- Il existe des différences statistiquement significatives entre le pré-test et le post-test dans le développement de la perception mentale dans les activités sportives physiques des collégiens.

- Il existe des différences statistiquement significatives entre le pré-test et le post-test dans le développement de la capacité à se détendre dans les activités sportives physiques collectives chez les élèves du secondaire

- Il existe des différences statistiquement significatives entre le pré-test et le post-test dans le développement de la capacité à faire face à l'anxiété dans les activités sportives physiques des collégiens.

- Il existe des différences statistiquement significatives entre le pré-test et le post-test dans le développement de la motivation de la réussite sportive dans les activités sportives physiques collectives chez les collégiens.

- Il existe des différences statistiquement significatives entre le pré-test et le post-test dans le développement de la capacité à se mettre d'accord sur les activités sportives physiques des collégiens.

\section{Objectifs de recherche}

- Connaître l'ampleur de l'effet de l'activité physique dans les sports collectifs sur le développement de la perception mentale des élèves

- Déterminer dans quelle mesure l'activité physique physique influe sur le développement de la capacité des élèves à se détendre

- Apprendre à quel point l'activité physique affecte le développement de la capacité de copie chez les élèves

- Connaître le montant auquel l'activité physique du sport collectif dans le développement de la motivation de la réussite sportive des élèves

- Déterminer dans quelle mesure l'activité physique en groupe influe sur le développement de la concentration de l'attention chez les élèves

- Pour voir comment l'activité physique collective affecte le développement de la confiance en soi. 


\section{5- Méthodologie de recherche:}

Dans cette étude, nous nous sommes appuyés sur l'approche descriptive, qui est pertinente pour le sujet de notre recherche.

\section{Communauté de Recherche:}

La communauté d'origine est composée de 10000 étudiants et d'un étudiant.

\section{6-1- Échantillon de recherche et méthode de sélection:}

Après avoir ajusté les variables en menant l'étude exploratoire, l'échantillon de la recherche a été choisi de manière délibérée et est de $40 \%$. L'étudiant équivaut à $11 \%$ de la communauté d'origine.

\begin{tabular}{|l|c|c|}
\hline Dimensions & Positive statement numbers & Negative statement numbers \\
\hline possibilité de visualiser & $1,7,19$ & 13 \\
\hline La capacité de se détendre & $2,14,20$ & 8 \\
\hline La capacité de focaliser l'attention & & $3,9,15,21$ \\
\hline La capacité de faire face à l'anxiété & & $4,10,16,22$ \\
\hline Confiance en soi & 5,17 & 11,23 \\
\hline Réalisations sportives & & $6,12,18,24$ \\
\hline
\end{tabular}

Lors de la correction de l'échelle, des scores sont attribués aux expressions dans le sens de la dimension positive en fonction du niveau défini par le joueur devant chaque énoncé. En outre, les scores indiqués par le joueur sont inversés en termes de négatifs $1=6,2=5,4=3,5=2,6=1$.

Les scores de chaque dimension sont collectées séparément et plus le score du joueur du plus haut degré et la valeur de 24 dans chaque dimension comme indiqué par la caractéristique mesurée par cette dimension, et les degrés du joueur bas, comme en témoigne la nécessité de acquérir plus de formation sur les compétences mentales qui Mesuré par cette dimension.

Le nombre (1) indique que la phrase ne s'applique pas à vous complètement, le nombre (2) indique que l'expression s'applique à vous très peu, le nombre (3) à un petit degré, le nombre (4)) À un très dans une large mesure.

\section{6-2- Fondements scientifiques de l'échelle:}

Le Questionnaire sur les compétences mentales a été conçu par (Bull, John Albinson et Christopher Shambrook« 1992 »)traduit par( AkramZakiHatayebieh. (1996); pour mesurer certains des aspects mentaux importants de la performance athlétique:

La capacité de visualiser la capacité à se détendre Capacité à attirer l'attention capacité à faire face à l'anxiété Stimulation de soimême.L'intelligence comprend 24 mots et chacun des six extrêmes représentés par 3 déclarations. L'athlète répond à l'échelle de six points 
(s'applique à moi beaucoup, très, modérément, très peu, ne s'applique pas à moi complètement).

\section{7-Transactions scientifiques:}

\section{7-1-Stabilité:}

Les coefficients de stabilité ont été trouvés en appliquant l'échelle et en la réappliquant après trois semaines et en utilisant le coefficient alpha. Ceux-ci variaient entre 0,65 et 0,91 lorsqu'ils étaient appliqués à de multiples échantillons d'athlètes participant à de multiples activités sportives.

\section{7-2-Honnêteté:}

La véracité de la pointe a été vérifiée lorsque l'intelligence a été appliquée en conjonction avec certaines mesures qui mesurent des dimensions similaires.

\section{Stabilité:}

Le coefficient de stabilité a été calculé en fonction de l'échantillon de l'étude, qui comprend 10 étudiants utilisant la méthode à mi-chemin

Tableau 02: Le degré de stabilité de l'échelle appliquée aux élèves de la classe d'éducation physique et sportive.

\begin{tabular}{|c|c|c|c|c|c|c|}
\hline $\begin{array}{l}\text { Statistical } \\
\text { significance }\end{array}$ & $\begin{array}{l}\text { Level of } \\
\text { significance }\end{array}$ & $\begin{array}{l}\text { Degree of } \\
\text { freedom }\end{array}$ & $\begin{array}{l}\mathrm{R} \\
\text { Scheduled }\end{array}$ & $\begin{array}{l}\mathrm{R} \\
\text { Calcul }\end{array}$ & $\begin{array}{l}\text { sample } \\
\text { Scientific basis }\end{array}$ & $\begin{array}{l}\text { D. } \\
\text { Statistically }\end{array}$ \\
\hline Stability & 10 & 9 & 0.60 & 0.92 & 0.05 & D.S \\
\hline
\end{tabular}

Notez que la valeur de coefficient de corrélation calculée: 0.92 est supérieure à la valeur de consigne, que nous avons trouvée égale à 0.60 au degré de liberté de 9 et au niveau de signification de 0.05 , donc l'échelle a une grande stabilité

Vérification: La validité du questionnaire consiste à s'assurer qu'il mesurera ce qui a été préparé pour le mesurer.

Il est également prévu d'inclure le questionnaire pour tous les éléments qui doivent être inclus dans l'analyse d'une part, et la clarté des paragraphes et de leur vocabulaire d'autre part, afin qu'ils soient compris par tous ceux qui les utilisent.

6.4 Les arbitres ont été certifiés: l'échelle a été montrée aux professeurs pour un avis où la validité de l'outil a été approuvée pour les hypothèses de recherche.

\section{6-5- Authentifier les statistiques:}

Calculer la vérité statistique à partir de la racine carrée du coefficient de stabilité. 
Le coefficient de stabilité est de 0,92 et la racine est la constante de stabilité et est égale à 0,95 .

Notez que le coefficient d'honnêteté est de 0,95 et cela indique que l'échelle est très honnête.

Présentation, analyse et comparaison des résultats avec des hypothèses:

\section{Présentation et analyse de la première hypothèse qui stipule:}

Les différences statistiques de pré-test et post-test de l'activité physique de sport collectif dans le développement de la concentration de l'attention chez les collégiens.

Échantillon Écart-type Moyenne arithmétique Degré de liberté Tablar

(T) (T) Calcul de la signifiance du niveau de signifiance

Tableau (03): montre la valeur de T pour indiquer les différences entre le pré-test et le posttest (concentration d'attention).

\begin{tabular}{|l|c|c|c|c|c|c|c|c|}
\hline Zones & Echantillon & $\begin{array}{l}\text { Standard } \\
\text { Deviation }\end{array}$ & $\begin{array}{l}\text { Arithmetic } \\
\text { mean }\end{array}$ & $\begin{array}{l}\text { Degree of } \\
\text { Freedom }\end{array}$ & $\begin{array}{l}\text { Tablar } \\
(\mathbf{T})\end{array}$ & (T)Calculâtes & $\begin{array}{l}\text { signifiance } \\
\text { level }\end{array}$ & signifiance \\
\hline Test tribal & 140 & 3.47 & 17.43 & 139 & 1.68 & 2.93 & 0.05 & $\mathrm{D}$ \\
\hline Post-test & 140 & 4.20 & 18.75 & & & & & $\mathrm{D}$ \\
\hline
\end{tabular}

La moyenne arithmétique du test tribal est estimée. Avec un écarttype de 3,47 et la moyenne du post-test était de 18,75 avec un écart-type de 4,20 . En termes de $T$ calculé, il était de 2,93 , ce qui est supérieur à la valeur traditionnelle de 1,68. - À-dire, au niveau de « $0,05=» »$, et le degré de liberté 139 , ce qui indique qu'il existe des différences statistiquement significatives entre le pré-test et post-test à se concentrer l'attention dans les sports collectifs, ce qui est ce que je suis arrivé

Puisque le «T» calculé est supérieur au «T», cela indique un écart entre le pré-test et le post-test pour attirer l'attention sur les élèves dans les sports de groupe.

\section{7-1- Discussion de la première hypothèse :}

Les résultats indiqués dans le tableau (03) montrent qu'il existe des différences dans le pré-test et post-test, où la valeur calculée «T $\mathrm{T} »\langle 2,93$ » et « $\mathrm{T}$ » tableau « 1.68 » et donc depuis le calcul « $\mathrm{T}$ » est supérieure à la « $\mathrm{t}$ » prévu, nous disons que la première hypothèse a été réalisée sur la base des résultats et c'est la conclusion d'une étude indique Mohammed Hassan Allaoui que les athlètes ont une difficulté claire dans la capacité à concentrer l'attention à la lumière de ce fait, l'athlète devrait réduire la pression psychologique grave Il peut tomber sur les épaules du sport parce qu'il travaille à réduire la capacité de l'athlète à se concentrer.

« Oussama Kamel Rateb » a souligné que la concentration de l'attention est l'une des compétences psychologiques qui sont importants pour le succès 
$\mathrm{du}$ processus de l'éducation, la formation ou la concurrence dans ses différentes formes, détourné l'attention ou manque de concentration, ce qui affecte négativement la performance ( O samarateb ;2001).

Mohammed Al-Arabi Al-Shammoun souligne également que l'apparition de réalisations mathématiques est liée à la présence de l'athlète dans une énergie psychique optimale. Sa caractéristique la plus importante est que l'attention a été entièrement dirigée vers des performances habiles et que l'attention est une dimension essentielle dans l'entraînement et la compétition à tous les niveaux (al-shammoun ;1998).

\subsection{Présentation et analyse de la deuxième hypothèse qui stipule:}

Différences statistiques de pré-test et post-test de l'activité physique de sport collectif dans le développement de la perception mentale chez les élèves du collège Échantillon Écart-type Moyenne arithmétique Degré de liberté Tablar (T) (T) Calcul de la signifiance du niveau de signifiance.

Tableau (04): montre la valeur de $\mathrm{T}$ pour indiquer les différences entre le pré-test et le posttest dans la perception mentale.

\begin{tabular}{|l|l|l|l|l|l|l|l|l|}
\hline Zones & Echantillon & $\begin{array}{l}\text { Standard } \\
\text { Deviation }\end{array}$ & $\begin{array}{l}\text { Arithmetic } \\
\text { mean }\end{array}$ & $\begin{array}{l}\text { Degree of } \\
\text { Freedom }\end{array}$ & $\begin{array}{l}\text { Tablar } \\
(\mathbf{T})\end{array}$ & (T)Calculâtes & $\begin{array}{l}\text { signifiance } \\
\text { level }\end{array}$ & signifiance \\
\hline $\begin{array}{l}\text { Test } \\
\text { tribal }\end{array}$ & 140 & 3.59 & 17.05 & 139 & 1.68 & 1.75 & 0.05 & D \\
\hline $\begin{array}{l}\text { Post- } \\
\text { test }\end{array}$ & 140 & 3.40 & 17.77 & & & & & D \\
\hline
\end{tabular}

La moyenne arithmétique du test tribal est estimée. 17,77 « avec un écart-type de » 3,59 « et la moyenne pour le post-test a été estimé à »17.05 « avec un écart type de »3,40 «tandis que pour la valeur T calculée est »1,75 « qui est supérieure à la valeur de le traditionnel $\mathrm{T}$ "1,68" "0,05 =" "et le degré de liberté 139, une valeur indiquant qu'il existe des différences statistiquement significatives entre pré-test et post-test de perception mentale dans les sports collectifs

\section{7-3- Discussion de la deuxième hypothèse;}

Les résultats indiqués dans le tableau (04) montrent qu'il existe des différences dans le pré-test et post-test, où la valeur calculée « $\mathrm{T}$ » est la différence entre le pré-test et post-test (perception mentale). «1,75» et « T» tableau « 1.68 » et donc depuis le « $\mathrm{T}$ » calculée supérieure à la « $\mathrm{T}$ » prévu nous disons que la seconde hypothèse a été réalisée sur la base des résultats et c'est la conclusion de l'étude de Mohammed Allaoui résumé les différentes utilisations de la perception mentale dans les sports exemples d'applications pratiques:

À partir de là, nous concluons que l'hypothèse selon laquelle il existe des différences statistiquement significatives entre le pré-test et le post-test dans la perception mentale des collégiens a été réalisée. 


\subsection{Présentation et analyse de la troisième hypothèse, qui stipule que:}

Les différences statistiques de pré-test et post-test de l'activité physique de sport collectif dans le développement de la capacité à se détendre chez les élèves du collège Échantillon Écart-type Moyenne arithmétique Degré de liberté Tablar (T) (T) Calcul de la signifiance du niveau de signifiance. La moyenne arithmétique du test tribal est significative. Avec un écart-type de 4,67 et un tapis.

Tableau (06): affiche la valeur de (T) pour indiquer les différences entre le pré-test et le post-test dans le développement de la capacité à se détendre.

\begin{tabular}{|l|l|l|l|l|l|l|l|l|}
\hline Zones & Echantillon & $\begin{array}{l}\text { Standard } \\
\text { Deviation }\end{array}$ & $\begin{array}{l}\text { Arithmetic } \\
\text { mean }\end{array}$ & $\begin{array}{l}\text { Degree of } \\
\text { Freedom }\end{array}$ & $\begin{array}{l}\text { Tablar } \\
(\mathbf{T})\end{array}$ & (T)Calculâtes & $\begin{array}{l}\text { signifiance } \\
\text { level }\end{array}$ & signifiance \\
\hline $\begin{array}{l}\text { Test } \\
\text { tribal }\end{array}$ & 140 & 4.67 & 15.50 & 139 & 1.68 & 3.20 & 0.05 & D \\
\hline $\begin{array}{l}\text { Post- } \\
\text { test }\end{array}$ & $\mathbf{1 4 0}$ & $\mathbf{3 . 6 5}$ & $\mathbf{1 7 . 0 7}$ & & & & & D \\
\hline
\end{tabular}

\subsection{Présentation et analyse de la troisième hypothèse, qui stipule que:}

Les différences statistiques de pré-test et post-test de l'activité physique de sport collectif dans le développement de la capacité à se détendre chez les élèves du collège Échantillon Écart-type Moyenne arithmétique Degré de liberté Tablar (T) (T) Calcul de la signifiance du niveau de signifiance.

Tableau (06): affiche la valeur de T pour indiquer les différences entre le pré-test et le posttest dans le développement de la capacité à se détendre.

\begin{tabular}{|l|l|l|l|l|l|l|l|l|}
\hline Zones & Echantillon & $\begin{array}{l}\text { Standard } \\
\text { Deviation }\end{array}$ & $\begin{array}{l}\text { Arithmetic } \\
\text { mean }\end{array}$ & $\begin{array}{l}\text { Degree } \\
\text { of } \\
\text { Freedom }\end{array}$ & $\begin{array}{l}\text { Tablar } \\
(\mathbf{T})\end{array}$ & (T)Calculâtes & $\begin{array}{l}\text { signifiance } \\
\text { level }\end{array}$ & signifiance \\
\hline $\begin{array}{l}\text { Test } \\
\text { tribal }\end{array}$ & 140 & 4.61 & 15.45 & 139 & 1.68 & 4.78 & 0.05 & D \\
\hline $\begin{array}{l}\text { Post- } \\
\text { test }\end{array}$ & 140 & 3.87 & 17.84 & & & & & D \\
\hline
\end{tabular}

La moyenne arithmétique du test tribal est significative. Avec un écart type de 4,67 et une moyenne mathématique de 17,07 avec un écart type de 3,65 et une valeur calculée de 3,20, qui est supérieure à la valeur classique de 1,68 , soit au niveau de signification « $0,05=» »$, et la degré de liberté 139, une valeur indiquant qu'il existe des différences statistiquement significatives entre pré-test et post-test dans le développement de la capacité à se détendre dans les sports collectifs

Puisque le «T» calculé est supérieur au «T», cela indique qu'il y a une différence entre le pré-test et le post-test dans le développement de la capacité à détendre les élèves dans les sports collectifs. 


\section{5- Discussion de la troisième hypothèse:}

Les résultats révélés dans le tableau (06), où calculé « $\mathrm{T} »$, « $3.20 »$, et « $\mathrm{T}$ » Le tableau « 1.68 » et donc depuis le calcul « $\mathrm{T}$ » est supérieure à la « $\mathrm{t}$ » qui est prévu, nous disons que la troisième hypothèse a été réalisée

Sur la base des résultats et c'est ce que l'étude de Nat et Solin que la relaxation aide à développer des aspects physiologiques, physiques et psychologiques, et d'augmenter la capacité de travailler dans de longues périodes et de réduire la fatigue et la capacité de détendre les muscles.

\section{7-6- Présentation et analyse de la quatrième hypothèse qui stipule que:}

Différences de signification statistique pour le pré-test et le post-test des activités sportives physiques collectives dans le développement de la capacité d'adaptation des collégiens Échantillon Écart-type Moyenne arithmétique Degré de liberté Tablar (T) (T) Calcul de la signifiance du niveau de signifiance.

Tableau (07): montre la valeur de T pour indiquer les différences entre le pré-test et le posttest dans le développement de la capacité de copie.

\begin{tabular}{|l|l|l|l|l|l|l|l|l|}
\hline Areas & Sample & $\begin{array}{l}\text { Standard } \\
\text { Deviation }\end{array}$ & $\begin{array}{l}\text { Arithmetic } \\
\text { mean }\end{array}$ & $\begin{array}{l}\text { Degree of } \\
\text { Freedom }\end{array}$ & $\begin{array}{l}\text { Tablar } \\
(\mathbf{T})\end{array}$ & (T)Calculâtes & $\begin{array}{l}\text { signifiance } \\
\text { level }\end{array}$ & signifiance \\
\hline $\begin{array}{l}\text { Tribal } \\
\text { test }\end{array}$ & 140 & 3.19 & 17.43 & 139 & 1.68 & 5.07 & 0.05 & D \\
\hline $\begin{array}{l}\text { Post- } \\
\text { test }\end{array}$ & 140 & 4.20 & 18.75 & & & & & D \\
\hline
\end{tabular}

La moyenne arithmétique du test tribal est estimée. 15,45 « avec un écart type de 4,61 » et la moyenne mathématique pour le post-test a été estimé à 17,84 avec un écart type de 3,87 et pour la valeur T calculée était de 4,78 , qui est supérieure à la valeur initiale de $\mathrm{T} 1,68$, soit à le niveau de signification « $0,05=» »$, et le degré de liberté 139 , une valeur indiquant qu'il existe des différences statistiquement significatives entre les pré-test et post-test dans le développement de la capacité de faire face à l'anxiété dans les sports collectifs

Puisque le «T» calculé est supérieur au «T», cela indique qu'il existe une différence entre le pré-test et le post-test pour développer la capacité d'adaptation des élèves dans les sports collectifs.

\section{7-7- Discussion de la quatrième hypothèse:}

Les résultats ont montré dans le tableau (07) où la valeur calculée « T » est 4,78 « et » $\mathrm{T}$ 《» Tableau 1.68 « Ainsi, étant donné que la valeur calculée » $\mathrm{T}$ 《 est supérieure à la » $\mathrm{T}$. «nous disons que la quatrième hypothèse a été atteint en se fondant sur les résultats, ce qui est le résultat de l'étude de » Savoie C », ce qui indique qu'il est nécessaire d'aborder l'anxiété et le travail pour réduire l'intensité et ouvre la voie à augmenter l'attention se 
concentrer et ainsi obtenir les meilleurs résultats du sport et est par le biais du programme de formation mentale et représente la motivation la motivation personnelle à jouer le jeu et la lutte pour réussir l'excellence et de l'excellence et est donc une mesure de gravité par les athlètes pour atteindre un haut niveau.

Ceci est cohérent avec les résultats des études de Hassan Abou Abia, qui a souligné que l'anxiété est l'un des plus importants phénomènes psychologiques associés à l'organisation de compétitions sportives, où il joue un rôle important en influençant la performance des athlètes, en particulier les jeunes personnes, en raison de leurs aspirations à gagner le résultat des totems et ne pas perdre (Hassan.2003).

\section{7-8- Présentation et analyse de la cinquième hypothèse qui stipule que:}

Différences de signification statistique pour le pré-test et le post-test des activités sportives physiques collectives dans le développement de la motivation de réalisation mathématique des élèves du secondaire Échantillon Écart-type Moyenne arithmétique Degré de liberté Tablar (T) (T) Calcul de la signifiance du niveau de signifiance.

Tableau (08): montre la valeur de T pour indiquer les différences entre le pré-test et le posttest dans le développement de la motivation pour l'accomplissement sportif.

\begin{tabular}{|l|l|l|l|l|l|l|l|l|}
\hline Zones & Echantillon & $\begin{array}{l}\text { Standard } \\
\text { Deviation }\end{array}$ & $\begin{array}{l}\text { Arithmetic } \\
\text { mean }\end{array}$ & $\begin{array}{l}\text { Degree of } \\
\text { Freedom }\end{array}$ & $\begin{array}{l}\text { Tablar } \\
(\mathbf{T})\end{array}$ & (T)Calculâtes & $\begin{array}{l}\text { signifiance } \\
\text { level }\end{array}$ & signifiance \\
\hline $\begin{array}{l}\text { Test } \\
\text { tribal }\end{array}$ & 140 & 4.66 & 17.80 & 139 & 1.68 & 1.88 & 0.05 & D \\
\hline $\begin{array}{l}\text { Post- } \\
\text { test }\end{array}$ & 140 & 4.24 & 18.78 & & & & & D \\
\hline
\end{tabular}

La moyenne arithmétique du test tribal est estimée. 17,43 "avec un écart-type de 3,19" et la moyenne pour le post-test était de 18,75 avec un écart-type

\section{7-8- Présentation et analyse de la cinquième hypothèse qui stipule que:}

Différences de signification statistique pour le pré-test et le post-test des activités sportives physiques collectives dans le développement de la motivation de réalisation mathématique des élèves du secondaire Échantillon Écart-type Moyenne arithmétique Degré de liberté Tablar (T) (T) Calcul de la signifiance du niveau de signifiance. 
Tableau (08): montre la valeur de $(\mathrm{T})$ pour indiquer les différences entre le pré-test et le post-test dans le développement de la motivation pour l'accomplissement sportif.

\begin{tabular}{|l|l|l|l|l|l|l|l|l|}
\hline Zones & Echantillon & $\begin{array}{l}\text { Standard } \\
\text { Deviation }\end{array}$ & $\begin{array}{l}\text { Arithmetic } \\
\text { mean }\end{array}$ & $\begin{array}{l}\text { Degree } \\
\text { of } \\
\text { Freedom }\end{array}$ & $\begin{array}{l}\text { Tablar } \\
(\mathbf{T})\end{array}$ & (T)Calculâtes & $\begin{array}{l}\text { signifiance } \\
\text { level }\end{array}$ & signifiance \\
\hline $\begin{array}{l}\text { Test } \\
\text { tribal }\end{array}$ & 140 & 4.66 & 17.80 & 139 & 1.68 & 1.88 & 0.05 & D \\
\hline $\begin{array}{l}\text { Post- } \\
\text { test }\end{array}$ & 140 & 4.24 & 18.78 & & & & & D \\
\hline
\end{tabular}

La moyenne arithmétique du test tribal est estimée. 17,43 « avec un écart type de 3,19 » et la moyenne pour le post-test était de 18,75 avec un écart type de 4,20 et la valeur calculée de « 5,07» « $0,05=» »$, et le degré de liberté 139 , une valeur indiquant qu'il existe des différences statistiquement significatives entre le pré-test et le post-test dans le développement de la motivation pour la réussite dans les sports collectifs sportifs.

Puisque le «T» calculé est supérieur au «T», cela indique qu'il y a une différence entre le pré-test et le post-test dans le développement de la motivation pour la réussite athlétique chez les étudiants dans les sports collectifs.

\section{7-9 Discussion de la cinquième hypothèse}

Les résultats indiqués dans le tableau (08) montrent qu'il existe des différences entre le pré-test et le post-test, où le «T» calculé est la différence entre le pré-test et le post-test (perception mentale). "5.07" et "T" tabulaire "1.68" et puisque puisque le "T" est supérieur au " $t$ ", on dit que l'hypothèse

Les résultats de cette étude ont été comparés à l'étude de (Mohiuddin Hussein 1988), qui a montré que la motivation de la réussite dans certaines sociétés, même celles que l'on appelle sociétés avancées, diminue.

(Mohiuddin Hussein 1971) ajoute qu'il y a un certain nombre de raisons derrière le déclin de la motivation chez les étudiants universitaires. Ces dernières années, il y a eu un changement dans les rôles de la famille pendant de longues périodes en raison de leur préoccupation pour le travail et les enfants ont un sentiment d'appartenance plus élevé que leur désir d'atteindre l'excellence. la motivation des enseignants à tomber au-dessous du niveau attendu d'eux et reflète le climat socio-psychologique fourni par la société en général, qui a affecté le développement des compétences mentales en particulier.(Mohiuddin Hussein ,1971).

Nous concluons que l'hypothèse selon laquelle il existe des différences statistiquement significatives dans le pré-test et le post-test des activités physiques sportives collectives dans le développement de la réussite mathématique des élèves du secondaire a été réalisée 


\section{7-9 Présentation et analyse de la sixième hypothèse qui stipule:}

Différences statistiques pour le pré-test et le post-test des activités physiques sportives collectives dans le développement de la capacité d'adaptation chez les élèves du secondaire Échantillon Écart-type Moyenne arithmétique Degré de liberté Tablar (T) (T) Calcul de la signifiance du niveau de signifiance.

Tableau (09): montre la valeur de $\mathrm{T}$ pour indiquer les différences entre pré-test et post-test dans le développement de la capacité de copie.

\begin{tabular}{|l|l|l|l|l|l|l|l|l|}
\hline Zones & Echantillon & $\begin{array}{l}\text { Standard } \\
\text { Deviation }\end{array}$ & $\begin{array}{l}\text { Arithmetic } \\
\text { mean }\end{array}$ & $\begin{array}{l}\text { Degree } \\
\text { of } \\
\text { Freedom }\end{array}$ & $\begin{array}{l}\text { Tablar } \\
\text { (T) }\end{array}$ & (T)Calculâtes & $\begin{array}{l}\text { signifiance } \\
\text { level }\end{array}$ & signifiance \\
\hline $\begin{array}{l}\text { Test } \\
\text { tribal }\end{array}$ & 140 & 4.66 & 17.80 & 139 & 1.68 & 1.88 & 0.05 & D \\
\hline $\begin{array}{l}\text { Post- } \\
\text { test }\end{array}$ & 140 & 4.24 & 18.78 & & & & & D \\
\hline
\end{tabular}

La moyenne arithmétique du test tribal est estimée. Avec un écarttype de 4,66 et une moyenne pour le post-test a été estimée à 18,78 avec un écart-type de 4,24. En ce qui concerne la valeur de T calculée, il était de 4,78 , qui est supérieure à la valeur initiale de $\mathrm{T} 1,68$, soit au niveau de signification « $0,05=»$ », et le degré de liberté 139 , ce qui indique qu'il existe des différences statistiquement significatives entre le pré-test et le post-test dans le développement de la capacité à affronter les sports collectifs.

Puisque le $\mathrm{T}$ calculé est supérieur au $\mathrm{T}$ prévu, cela indique un écart entre le pré-test et le post-test pour développer la capacité d'adaptation des élèves dans les sports de groupe.

\section{7-10 Discussion de la sixième hypothèse:}

Les résultats révélés dans le tableau (09) ont montré que les différences entre le pré-test et le post-test étaient les valeurs $\mathrm{T}$ pour l'importance des différences (dans le développement de la capacité de copie). Les valeurs calculées de "T" et "4.78" Le tableau "1.68". Puisque, puisque le " $t$ " calculé est supérieur au " $t$ ", on dit que la sixième hypothèse a été réalisée.

Sur la base des résultats et c'est ce qui atteint par l'étude d'Oussama Kamel Rateb que la confiance en soi signifie l'attente réaliste de l'athlète à atteindre le succès, la confiance en soi ne signifie pas ce qu'il espère faire de l'athlète, mais quelles sont les choses réelles qui attendait le travail de la confiance en soi trois types: la confiance en soi - la confiance Le manque de confiance, le succès, la victoire et l'échec font partie du sport. athlètes autoconfiance savent ce fait et d'agir avec beaucoup de réalisme, mais les athlètes qu'ils attribuent à la confiance en soi ont peur de l'échec tant qu'ils font leur peur plus facile Et puis Y. Le résultat est que les athlètes qui croient que ils ont peu à faire sont psychologiquement captifs à leurs perceptions négatives 
et prendre le chemin de la défaite et l'échec, pas le succès et la victoire sur l'adversaire, et que la faiblesse de la confiance affaiblit l'efficacité de la performance et conduit à l'apparition de l'anxiété et l'incertitude de l'objectif en plus de ce qui précède, ces athlètes peuvent se sentir insuffisants même avec la poursuite de la pratique et qu'ils ne peuvent pas faire le meilleur et la confiance en soi dans l'attente des résultats font plus disposés à faire et de donner à l'athlète, et plus attendre et devenir un athlète plus confiant et chaque fois que $\mathrm{Z}$ Cette faiblesse de son endurance et sa persévérance et sa détermination face à des obstacles qui neutralisent ou les problèmes rencontrés

Bien que les résultats de l'étude de mon ami Nure-EDDine Mohammed, qui a indiqué que la confiance en soi des compétences importantes dans le domaine du sport en raison de son impact sur les performances des athlètes et que la confiance de l'athlète dans ses capacités est une source importante de positif pour atteindre l'énergie mentale positive et tire le chercheur de ce déjà déclaré que l'athlète Forces (jeux individuels) ont une faiblesse dans la confiance en soi en raison du manque de participation à des tournois officiels ( Nure-EDDine Mohammed ;1995).

L'hypothèse générale que: Il existe des différences statistiquement significatives entre le pré-test et post-test dans le développement de certaines habiletés mentales dans les activités sportives physiques des élèves des écoles intermédiaires collectifs ont été atteints.

\section{8-Conclusion générale:}

Après avoir présenté, analysé et discuté les résultats obtenus à travers l'étude de terrain, nous constatons qu'il y a:

- différences statistiques significatives entre le pré-test et post-test dans le développement de la concentration de l'attention dans les activités sportives physiques des étudiants intermédiaires en faveur du posttest, ce qui indique que les sports collectifs ont un rôle dans le développement des habiletés mentales.

- différences statistiques significatives entre le pré-test et post-test dans le développement de la perception mentale dans les activités sportives physiques des étudiants collectifs dans l'étape intermédiaire en faveur du post-test, indiquant que les sports collectifs ont un rôle dans la développement de compétences mentales.

- différences statistiques significatives entre le pré-test et post-test dans le développement de la capacité de se détendre dans les activités sportives physiques collectives des étudiants intermédiaires en faveur du post-test, ce qui indique que les sports collectifs ont un rôle dans le développement de la santé mentale compétences. 
- différences statistiques significatives entre le pré-test et post-test dans le développement de la capacité de faire face à l'inquiétude dans les activités sportives physiques collectives des étudiants intermédiaires en faveur du post-test, ce qui indique que les sports collectifs ont un rôle dans le développement des compétences mentales.

- Il existe des différences statistiquement significatives entre le pré-test et post-test dans le développement de la motivation de succès en mathématiques dans les activités sportives physiques des étudiants intermédiaires en faveur du post-test, ce qui indique que les sports collectifs ont un rôle dans le développement des compétences mentales.

- Il existe des différences statistiquement significatives entre le pré-test et post-test dans le développement de la capacité à faire face aux activités physiques des élèves de collège en faveur du post-test indique que les sports collectifs ont un rôle dans le développement des habiletés mentales .

D'ici, on peut dire que les activités sportives collectives jouent un rôle majeur dans le développement des capacités mentales de l'adolescent, ce qui l'affecte positivement

\section{9-Futures propositions et hypothèses:}

Sur la base des résultats précédents, nous espérons que l'étude en cours sera une première étape pour la réalisation d'études futures afin d'identifier l'effet des facteurs mentionnés ci-dessus sur la détermination de la tendance positive ou des études complémentaires de l'étude en incluant des variables et des facteurs subjectifs lié à la direction que nous n'avons pas abordé dans cette étude physique dans le développement de certaines compétences mentales chez l'adolescent, qui a un rôle positif.

Enfin, nous présentons quelques suggestions scientifiques et pratiques qui, selon nous, sont très importantes pour cette tranche d'âge à travers la pratique sportive dans les établissements d'enseignement et pour bénéficier de ce que ce segment peut réaliser.

Pour bénéficier de l'aspect scientifique de cette recherche dans le processus de la formation de professeurs d'éducation physique et des sports et de voir les méthodes et les méthodes modernes dans ce domaine.

La nécessité de prêter attention aux activités physiques et sportives dans les établissements d'enseignement et de leur donner leur statut pédagogique que d'autres matériaux en utilisant les moyens modernes d'enseignement et qui contribuent à l'augmentation à l'activité sportive.

Mettre l'accent sur l'importance d'une programmation appropriée pour les cours d'éducation physique et sportive et donner une image claire de l'impact de l'activité physique sur le développement des aptitudes mentales. 
Une contribution efficace à l'expansion de l'amitié entre les étudiants en fonction de leur interaction avec les autres compétitions et des cours de sport.

L'élève a acquis confiance en lui et sécurité grâce à son sens de l'exercice de son rôle au sein de l'équipe.

Vous devriez profiter de l'énergie excédentaire des élèves, puis prendre en compte leur confort et leur confort, ainsi que leur attention à la nutrition et à une nutrition adéquate.

Les problèmes rencontrés par l'adolescent sont nombreux et trouvé sérieux et sa tendance à distinguer des autres est une proie facile à la déviation sociale, nous devons donc envisager le moyen idéal pour intégrer cet adolescent au sein de la communauté et régit l'exercice de la gestion collective des moyens de vider et de satisfaire les besoins sociaux des adolescents, ce qui atténue les problèmes de la jeunesse dans la société.

\section{References:}

1. Mohamed Hassan Allawi. (1995). The burning psychology of the player and the trainer. Cairo: The Book Center for Publishing.

2. Osama KamelRateb (2001).Train psychological skills in the field of sports. Cairo: Arab Thought House.

3. AkramZakiHatayebieh. (1996). Contemporary Curricula in Physical Education and Sports i. Amman Jordan: Arab Thought House.

4. Mohammed Al-ArabiShamoun. (1998). Mental training in the field of sports. Cairo: Arab Thought House.

5. Mohammed Hassan Allawi. (2003).Sports Training Science. Cairo: Dar Al-Fikr. Knowledge. I 12

6. Hussein .Fouad Al-Bahi and Saad Abdel Rahman. (1988).Statistical psychology and measurement of the human mind i. Cairo: The Egyptian Printing House Press.

7. Hussein .Fouad Al-Bahi. (1971).Calendar and Psychometric Measurement. Cairo: The Anglo-Egyptian Library.

8. Eddine Mohamed . (1995).Skills preparation for football players. Technical Radiation Library: Cairo. 